\title{
Non-specialized inpatient care provided by university hospitals in Japan
}

\author{
Shin-ichi Toyabe \\ Crisis Management Office, Niigata \\ University Hospital, Niigata City, Japan
}

\begin{abstract}
In Japan, all citizens are covered by the national insurance system in which universal free access to healthcare services is promised to everybody. Even in tertiary care university hospitals, considerable numbers of secondary care inpatients are supposed to be treated. We studied the mixed state of secondary care and tertiary care in university hospitals in Japan and its year-to-year trend. Based on the results of a national survey, we could statistically classify Japanese case-mix classification into 821 groups that are supposed to need tertiary care (group A) and 296 groups that are supposed to need secondary care (group B). Sixty percent of patients admitted to the university hospitals belonged to group A, and $25 \%$ belonged to group B. Despite of the implementation of government policies to differentiate functions of hospitals, there was no trend toward an increase in the rate of tertiary care and decrease in the rate of secondary care from 2003 to 2006. Patient behavior to seek tertiary care was simply influenced by distance from university hospital. However, behavior of patients to seek secondary care was significantly influenced by distance to adjacent general hospitals and seize of these hospitals.
\end{abstract}

Keywords: inpatient care, Japan, university hospitals, patient behavior

\section{Introduction}

In Japan, all citizens are covered by a compulsory national health insurance system. ${ }^{1-3}$ Patients seeking primary care can freely choose various kinds of health care providers, including clinics, general hospitals and even specialized hospitals or university hospitals, and patients who need secondary care are often transferred from primary care providers directly to specialized hospitals or university hospitals. Functional differences between hospitals and nursing homes for the elderly in Japan are also not clear, and this is one of the main reasons for the extraordinary long hospital stay in Japan. ${ }^{4,5}$ Therefore, university hospitals in Japan play many roles other than the role of a tertiary care hospital, including primary caregiver, secondary care provider and chronic care hospital for the elderly. However, the extent of the mixed state of care provided by university hospitals in Japan, especially in terms of inpatient care, is not clear.

The government has tried to address the issue of undifferentiated functions of health care providers. A system of fees for referrals was introduced in $1988 .{ }^{6}$ In 1992 , hospitals staffed and equipped for advanced medical care could earn extra revenue. In 1996, patients who visit large hospitals without referrals from other clinics or hospitals have had to pay an extra charge. ${ }^{7}$ A mandatory social long-term care insurance system was implemented in 2000, and hospital beds were classified into general beds for acute care and beds for long term care by revision of the Medical Law at 2001. ${ }^{8}$ However, it is not clear whether these policies have been effective in establishing functional differentiation of university hospitals.

We recently reported that the function of university hospitals in Japan is undifferentiated in terms of referrals to and from university hospitals. ${ }^{9}$ In this study, we tried to clarify to what extent Japanese university hospitals specialize in tertiary care. We used 
the Japanese version of inpatient case-mix classification (Diagnosis Procedure Combination, DPC), which contains information on severity and complexity of patient illness, to divide inpatients into those who need tertiary care and those who need secondary care. By using this classification, we showed the mixed state of secondary care and tertiary care in university hospitals quantitatively. We also analyzed the interaction between university hospitals.

\section{Materials and methods}

\section{Outline of Japanese case-mix}

\section{classification}

Diagnostic Procedure Combination (DPC) is a Japanese patient classification system used to group types of inpatients discharged from acute care hospitals. ${ }^{9}$ There were 2,347 case-mix groupings in the 2006 version of DPC. Acute care hospitals obtain a fixed per-diem amount of insurance reimbursement for each inpatient under the DPC system, and the amount was determined for each case-mix grouping. Each patient was assigned to only one DPC group according to the type of disease of the patient, including major diagnostic categories, International Classification of the Diseases (ICD-10) codes, and the presence or absence of coma, surgical operations, invasive treatments and comorbidities. The insurance reimbursement system using the DPC system started in 2003 in 80 university hospitals and two special function hospitals, and it was extended to 360 acute care hospitals (total of 180,000 beds) in 2006 .

\section{Classification of DPCs according to the level of care}

In this manuscript, we defined primary care as care provided by primary care physicians and hospital outpatient care. Secondary care was defined as inpatient care provided in hospitals after referral from primary care providers. Tertiary care was defined as highly specialized inpatient care provided to patients with complex and refractory health problems. Since there is no clear distinction between secondary care and tertiary care, we tried to differentiate these two categories of care by using the DPC classification. The classification contains information on severity and complexity of health problems affecting patients. When the proportion of patients belonging to a certain DPC was statistically higher in university hospitals than in general hospitals, we considered that the DPC was for tertiary care (group A), and when the proportion of the patients belonging to a DPC was statistically higher in general hospitals than in university hospitals, we considered that the DPC was for secondary care (group B). We used data from a national survey on the impact of introduction of DPC. ${ }^{10}$ The data are clinical summary data on patients discharged from several categories of hospitals in 2005 and 2006, and they include number of patients belonging to each DPC as well as their average length of stay. The first category consisted of two special function hospitals and all 80 university hospitals in Japan. The total number of patients discharged from these hospitals was 175,373 , and the average length of stay was 16.8 days. We considered this category of hospitals as representative of university hospitals. The second category of hospitals included 371 hospitals, 351 hospitals of which were general hospitals. The total number of patients discharged from the hospitals was 331,533, and the average length of stay was 14.9 days. We considered this category of hospitals as representative of general hospitals. We investigated whether the proportion of patients with each DPC is higher or lower in university hospitals than in general hospitals. The chi-square test and Fisher's exact test were used for the analyses. If the proportion of patients with a DPC was statistically higher in university hospitals than in general hospitals, we classified the DPC as group A, and if the proportion was statistically higher in general hospitals than in university hospitals, we classified the DPC as group B.

\section{Characteristics of each group of DPCs}

Since the above-mentioned classification of DPCs is based on care actually provided to each patient rather than the care that is necessary for patients, we validated the classification. We analyzed the characteristics of group A DPCs using multivariate logistic analysis. The dependent variable was whether a DPC belongs to group A (1) or not (0). Independent variables in the analysis were important elements of definitions of DPCs such as major diagnostic categories and presence or absence of coma, surgical operations, invasive treatments and comorbidities. Categorical variables were coded into dummy variables. The forward stepwise method was used to determine significantly associated variables. The classification of DPC was also validated by using Charlson's index scores. ${ }^{11,12}$ The scores were compared between the two DPC groups by using Wilcoxon's rank sum test.

\section{Year-to-year trend in proportion of each DPC group}

Next, we analyzed whether implementation of health policies to promote functional differentiation of university hospitals have actually resulted in specialization of university hospitals 
in terms of levels of care. Year-to-year changes in proportions of inpatients belonging to group A and group B DPCs were analyzed using data for all inpatients discharged from Niigata University Hospital during the period from 2003 to 2006. Niigata University Hospital has 810 inpatient beds ( 748 acute care beds) and 23 clinical departments. About ten thousand patients are admitted per year. Since the definition of DPCs has been updated annually, all inpatients were coded again by the 2006 version of the DPC system.

\section{GIS analysis of patient behavior in secondary care referrals}

We analyzed interaction between Niigata University Hospital and the neighboring general hospitals using geographic information system (GIS). We hypothesized that the strength of interaction was correlated with parameters that were represented by functions of hospital size and distance between the hospitals. ${ }^{13}$ We used number of acute care beds as the scale of the hospital. Suppose that there were $\mathrm{j}$ general hospitals and i regions with $2 \mathrm{~km}$ size square in Niigata Prefecture. The strength $P_{i}$ that attracts patients admitted to $j$ neighboring general hospitals, the strength $P_{\text {univ, } i}$ that attracts patients admitted to the university hospital, and the ratio of attractive strength $P R_{i}$ in mesh $i$ were defined as follows, depending on the distance-decay parameter: ${ }^{14-17}$

$$
\begin{aligned}
& P_{1, i}=\sum_{j}\left(1 / d_{i j}\right), P_{\text {univ } 1, i}=1 / d_{\text {univ }, i}, P R_{1, i}=P_{u n i v 1, i} / P_{1, i}, \\
& P_{2, i}=\sum_{j}\left(b_{j} / d_{i j}\right), P_{\text {univ } 2, i}=b_{u n i v} / d_{\text {univ }, i}, P R_{2, i}=P_{\text {univ } 2, i} / P_{2, i}, \\
& P_{3, i}=\sum_{j}\left(1 / \exp \left(d_{i j}\right)\right), P_{\text {univ } 3, i}=1 / \exp \left(d_{\text {univ }, i}\right), P R_{3, i} \\
& =P_{\text {univ } 3, i} / P_{3, i}, \\
& P_{4, i}=\sum_{j}\left(b_{j} / \exp \left(d_{i j}\right)\right), P_{\text {univ } 4, i}=b_{\text {univ }} / \exp \left(d_{\text {univ }, i}\right), P R_{4, i} \\
& P_{5, i}=\sum_{j}\left(1 / d_{i j}^{2}\right), P_{\text {univ } 5, i}=1 / d_{\text {univ }, i}^{2}, P R_{5, i}=P_{\text {univ } 5, i} / P_{5, i}, \\
& P_{6, i}=\sum_{j}\left(b_{j} / d_{i j}^{2}\right), P_{\text {univ }, i}=b_{\text {univ }} / d_{\text {univ }, i}^{2}, \text { and } P R_{6, i} \\
& =P_{u n i v 6, i} / P_{6, i},
\end{aligned}
$$

where $d_{i j}$ was distance between centroid of mesh $i$ and hospital $j$ which has $b_{j}$ acute care beds. In the case of the university hospital, $d_{u n i v, i}$ was the distance and $b_{\text {univ }}$ was number of acute beds. The distance between the two points was calculated by travel distance using road network information. ${ }^{18}$ Patient admission rate was calculated for each mesh by dividing the number of patients admitted to Niigata University Hospital from each mesh region during 2006 by the total number of patients admitted to any hospital from the mesh. The total number of patients admitted to any hospitals from the mesh was estimated from population data for Niigata Prefecture and data for inpatients treated in hospitals located in Niigata Prefecture. ${ }^{19}$ Since information on exact numbers of patients admitted from subareas was not available, the estimation was performed under the hypothesis that admission rate to hospitals was constant within the area of each municipal body. We then analyzed whether the patient admission rate in a mesh region $i$ were associated with the attractive strength defined variously $\left(P, P_{\text {univ }}\right.$ and $\left.P R\right)$. Pearson's correlation analysis was performed to analyze the variables that were significantly associated with patient admission rates among the 18 indexes of attractive strength. Differences between correlation coefficients were evaluated after adjusting with Bonferroni's correction. Multiple regression analysis by the forced entry method was used to determine the variables that were most closely associated with the patient admission rates.

In all statistics, a $\mathrm{p}$ value less than 0.05 was considered significant. All statistical analyses except spatial analyses were performed using SPSS 15.0J (SPSS Japan Inc., Tokyo, Japan). GIS analyses were performed using MapCall Standard 2.1 (Chuo group, Niigata, Japan) and ArcGIS 9.2 (ESRI Japan Inc., Tokyo, Japan).

\section{Results}

\section{Classification of secondary and tertiary care patients based on DPCs}

We tried to classify DPCs into two categories based on data from a 2006 national survey on the impact of introduction of the DPC system: DPCs that were dominant in university hospitals rather than in general hospitals (group A DPCs) and DPCs that were dominant in general hospitals rather than in university hospitals (group B DPCs). The chi-square test and Fisher's exact test revealed that 821 of 2,347 DPCs belonged to group A and 296 belonged to group B. Proportions of the two categories of DPCs in university hospitals and general hospitals are shown in Figure 1. A considerable portion of patients who needed secondary care were treated in university hospitals, while many patients who needed tertiary care were treated in general hospitals.

\section{Characteristics of each DPC group}

To analyze the characteristics of group A DPCs, multivariate logistic analysis with the forward stepwise selection method was performed. The dependent variable was whether each 
$\%$

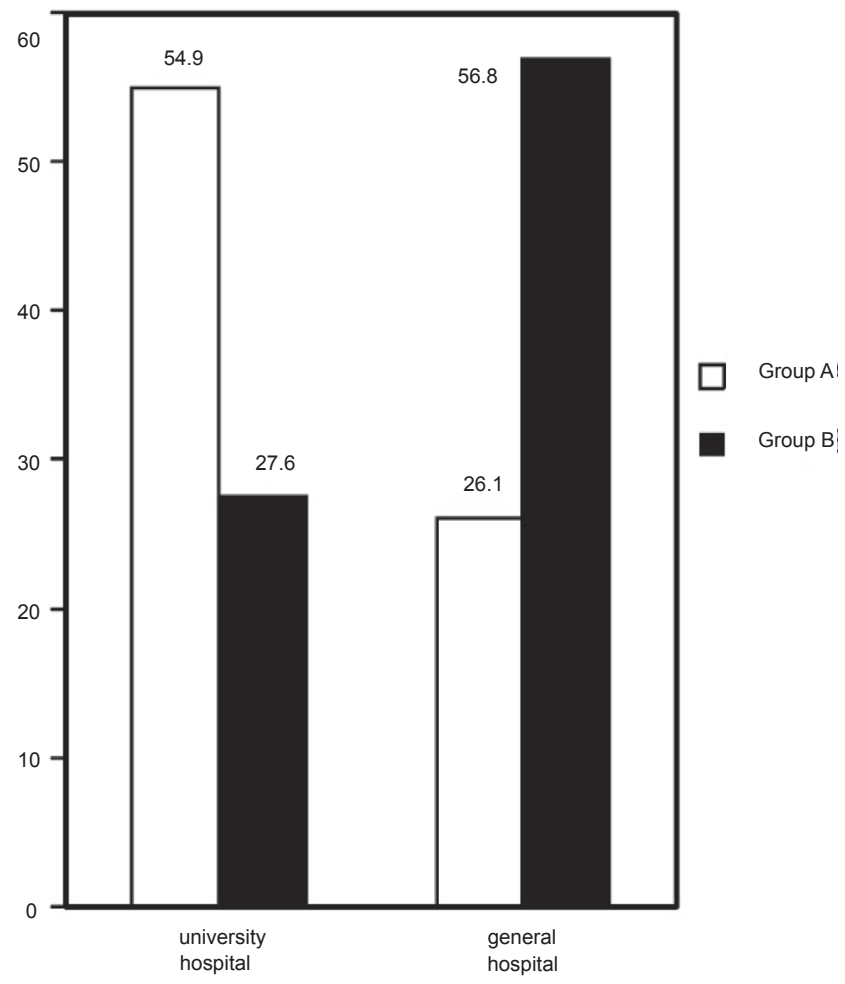

Figure I Proportions of the two Diagnostic Procedure Combination groups in university hospitals and general hospitals. The proportions of inpatients belonging to group A (open bars) and group B (shaded bars) in 82 hospitals (mainly university hospitals) and in 360 hospitals (mainly general hospitals) are shown.

DPC belongs to group A DPC (1) or not (0). The independent variables were important elements of the definition of DPCs. Table 1 shows the results of the analysis. DPCs with coma, surgical operations, invasive treatments and comorbidities are found to be dominant characteristics in group A. Charlson's scores of group A DPCs were significantly higher than that of group B DPC (Table 2, $\mathrm{p}<0.001$ ).

\section{Year-to-year trend in proportion of each DPC group}

We analyzed the extent to which university hospitals become more specialized as tertiary care hospitals in recent years. The time trend of the proportional change in each group of DPCs was analyzed using data for Niigata University Hospital from 2003 to 2006 as an example. As shown in Figure 2, the proportion of group A among total inpatients was about $60 \%$, whereas that of group B was about $25 \%$. The difference between the proportions of group A and group B in Niigata University Hospital was slightly larger than that of average data for all university hospitals (Figure 1).
However, there were no apparent time trends from 2003 to 2006 in the proportion of each group of DPCs.

\section{GIS analysis of patient behavior in secondary care referrals}

We carried out analysis to determine whether the distribution of inpatients admitted to Niigata University Hospital for secondary care was affected by neighboring general hospitals. We analyzed which decay parameter $\left(P, P r\right.$ and $\left.P_{u n i v}\right)$ was the most closely associated with the real distribution of inpatients belonging to each DPC group. Pearson's correlation analysis revealed that patient admission rate for group A DPCs was most strongly associated with attractive strength of Niigata University Hospital $P_{\text {univ } 2}(\mathrm{R}=0.458, \mathrm{p}<0.001)$ (Table 3, Figure $3 \mathrm{~A}$ ). On the other hand, patient admission rate for group B DPCs was most strongly associated with ratio of attractive strength of Niigata University Hospital to that of other general hospitals $P R_{2}(\mathrm{R}=0.606, \mathrm{p}<0.001)$ (Table 3, Figure $3 \mathrm{~B})$. Multiple regression analysis with forced entry of the two decay parameters into the equation revealed that admission rate of group A DPCs was associated with $P_{\text {univ } 2}$ and that admission rate of group B DPCs was associated with $P R_{2}$ (Table 4). These associations were obvious upon visual observation of geographic plots of these indices (Figure 4).

\section{Discussion}

In this study, we analyzed how and to what extent the care provided by university hospitals in Japan contains secondary care and whether the mixed state has been changed by the introduction of recent government policies. We revealed that tertiary care university hospitals in Japan provide secondary care to a considerable number of patients. Despite the recent implementation of government policies to promote functional differentiation of hospitals, there was no evidence of significant changes in the mixed state. Instead, it was found that university hospitals play a significant role as secondary care hospitals as do other general hospitals when secondary care referrals were required.

It is difficult to categorize care provided for inpatient care into secondary care and tertiary care in the Japanese setting because all citizens in Japan can freely access any hospitals in principle under the compulsory national health insurance system. A university hospital can be chosen as being a secondary care hospital by primary care providers, and a general hospital can also be chosen as being a tertiary care hospital by other general hospitals. In general, secondary care is usually defined as care given in a hospital after a referral from 
Table I Characteristics of group a diagnostic procedure combinations (DPCs)

\begin{tabular}{|c|c|c|c|c|}
\hline Items & B & SE & significance & $\operatorname{Exp}(B)$ \\
\hline \multicolumn{5}{|l|}{ Major diagnostic category (MDC) } \\
\hline Nervous system & 1.627 & 0.423 & $<0.001$ & 5.090 \\
\hline Eye & 3.977 & 0.759 & $<0.001$ & 53.359 \\
\hline Ear, nose, mouth and throat & 1.245 & 0.365 & 0.001 & 3.473 \\
\hline Digestive and hepatobiliary system & -1.037 & 0.242 & $<0.001$ & 0.354 \\
\hline Musculoskeletal system & 1.309 & 0.299 & $<0.001$ & 3.702 \\
\hline Skin and subcutaneous tissue & 2.623 & 0.601 & $<0.001$ & 13.784 \\
\hline Endocrine, nutrition and metabolic system & 2.288 & 0.478 & $<0.001$ & 9.859 \\
\hline Female reproductive system and pregnancy & 1.967 & 0.445 & $<0.001$ & 7.147 \\
\hline Blood and immunological disorders & 1.124 & 0.550 & 0.041 & 3.076 \\
\hline Newborn and other neonates & 4.010 & 0.743 & $<0.001$ & 55.129 \\
\hline \multicolumn{5}{|l|}{ Coma } \\
\hline without coma & -2.889 & 0.635 & $<0.001$ & 0.056 \\
\hline \multicolumn{5}{|l|}{ Surgical operation } \\
\hline with operation & 1.406 & 0.182 & $<0.001$ & 4.080 \\
\hline \multicolumn{5}{|l|}{ Invasive treatment (category $\# I$ ) } \\
\hline with treatment & 1.075 & 0.385 & 0.005 & 2.929 \\
\hline \multicolumn{5}{|l|}{ Invasive treatment (category \#2) } \\
\hline with treatment & 2.747 & 0.258 & $<0.001$ & 15.603 \\
\hline \multicolumn{5}{|l|}{ Comorbidities } \\
\hline without comorbidities & -1.195 & 0.324 & $<0.001$ & 0.303 \\
\hline with comorbidities & 1.769 & 0.568 & 0.002 & 5.866 \\
\hline
\end{tabular}

Multivariate logistic regression analysis with the forward stepwise selection method was performed to find characteristics of group A DPCs. The dependent variable was whether the DPC belongs to group A DPC (I) or not (0), and independent variables were important elements of the definition of DPC shown in the table.

a primary care provider, and tertiary care is advanced care delivered in specialized hospitals. However, the definitions of these levels of care are unclear in the Japanese setting. We tried to categorize these two levels of care based on the case-mix classification (DPC) assigned to each patient. It has been reported that there were differences in the rarity

Table 2 Frequency table of Charlson scores by diagnostic procedure combination (DPC) group.

\begin{tabular}{lll}
\hline Charlson's score & \multicolumn{2}{l}{ DPC frequency (\%) } \\
\cline { 2 - 3 } & group A & group B \\
\hline 0 & 77.4 & 63.4 \\
1 & 14.5 & 6.0 \\
2 & 0.3 & 1.3 \\
3 & 7.8 & 28.6 \\
4 & 0.0 & 0.0 \\
5 & 0.0 & 0.0 \\
$\geq 6$ & 0.0 & 0.7 \\
total & 100.0 & 100.0 \\
\hline
\end{tabular}

and complexity of diseases of patients in teaching hospitals and those in general hospitals in Japan. ${ }^{18}$ In agreement with this finding, statistical analyses revealed clear differences between dominant DPCs in university hospitals and general hospitals. By using these statistically dominant DPCs, we categorized patients into those who needed tertiary care (group A) and those who needed secondary care (group B). The categorization seems valid, since DPCs dominant in university hospitals consisted of diseases with severe and complicated clinical courses compared with DPCs that were not dominant in university hospitals. The patients belonging to group A DPCs had coma, received surgical operations and invasive treatments or had complications and comorbidities. In addition, the patients belonging to group A DPCs constituted a large portion of inpatients in university hospitals and the patients belonging to group B DPCs accounted for a large portion of inpatients in general hospitals.

In the mixed situation of both secondary care and tertiary care being provided by university hospitals, we speculate that university hospitals and general hospitals are competitive 


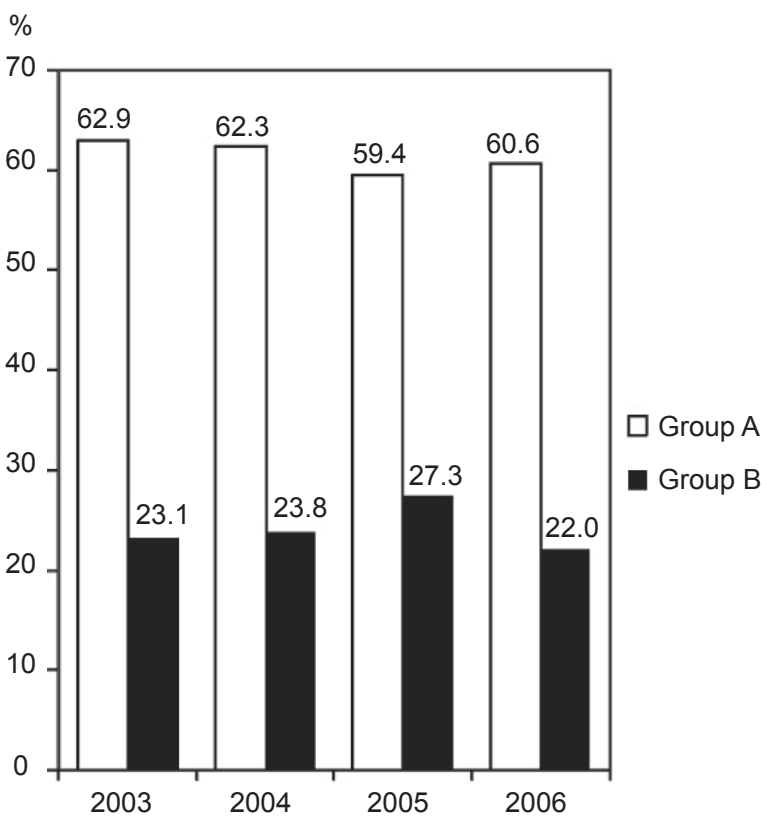

Figure 2 Year-to-year trend in the proportions of the two Diagnostic Procedure Combination groups. The proportions of inpatients belonging to group A (open bars) and group B (shaded bars) in Niigata University Hospital from 2003 to 2006 are shown.

Table 3 Pearson's correlation coefficients between admission rates and attractive strength parameters

\begin{tabular}{|c|c|c|c|c|}
\hline \multirow{3}{*}{$\begin{array}{l}\text { Attractive } \\
\text { strength } \\
\text { parameter }\end{array}$} & \multicolumn{4}{|c|}{ Admission rates } \\
\hline & \multicolumn{2}{|c|}{ Group A patients } & \multicolumn{2}{|c|}{ Group B patients } \\
\hline & $\overline{\mathbf{R}}$ & sig & $\overline{\mathbf{R}}$ & sig \\
\hline$\overline{P_{1}}$ & 0.328 & $<0.001$ & 0.496 & $<0.001$ \\
\hline$P_{\text {univl }}$ & 0.435 & $<0.001$ & 0.477 & $<0.001$ \\
\hline$P R_{l}$ & 0.365 & $<0.001$ & 0.584 & $<0.001$ \\
\hline$P_{2}$ & 0.291 & $<0.001$ & 0.422 & $<0.001$ \\
\hline$P_{\text {univ2 }} * 1$ & 0.458 & $<0.001$ & 0.477 & $<0.001$ \\
\hline$P R_{2} * 2$ & 0.328 & $<0.001$ & 0.606 & $<0.001$ \\
\hline$P_{3}$ & 0.266 & $<0.00$ I & 0.295 & $<0.001$ \\
\hline$P_{\text {univ3 }}$ & 0.394 & $<0.001$ & 0.489 & $<0.001$ \\
\hline$P R_{3}$ & 0.358 & $<0.001$ & 0.518 & $<0.001$ \\
\hline$P_{4}$ & 0.270 & $<0.00$ I & 0.300 & $<0.001$ \\
\hline$P_{\text {univ4 }}$ & 0.395 & $<0.00$ I & 0.489 & $<0.001$ \\
\hline$P R_{4}$ & 0.358 & $<0.001$ & 0.516 & $<0.001$ \\
\hline$P_{5}$ & 0.113 & 0.005 & 0.149 & $<0.001$ \\
\hline$P_{\text {univs }}$ & 0.321 & $<0.001$ & 0.228 & $<0.001$ \\
\hline$P R_{5}$ & 0.137 & 0.001 & 0.491 & $<0.001$ \\
\hline$P_{6}$ & 0.123 & 0.002 & 0.190 & $<0.001$ \\
\hline$P_{\text {unive }}$ & 0.381 & $<0.001$ & 0.228 & $<0.001$ \\
\hline$P R_{6}$ & 0.137 & 0.001 & 0.565 & $<0.001$ \\
\hline
\end{tabular}

The correlation coefficient between admission rate for group $A$ patients and $P_{\text {univ } 2}$ was statistically higher than the correlation coefficients between admission rate for group $A$ patients and $P_{2}, P_{3}, P_{4}, P_{5}, P R_{5}, P_{6}$ and $P R_{6}$, respectively $(* I)$. The correlation coefficient between admission rate for group $B$ patients and $P R_{2}$ was statistically higher than the correlation coefficients between admission rate for group $B$ patients and $P_{2}, P_{3}, P_{4}, P_{5}$, Puniv $_{5}, P_{6}$ and Puniv ${ }_{6}$, respectively $(* 2)$.

\section{A Admission rate of group A patients}

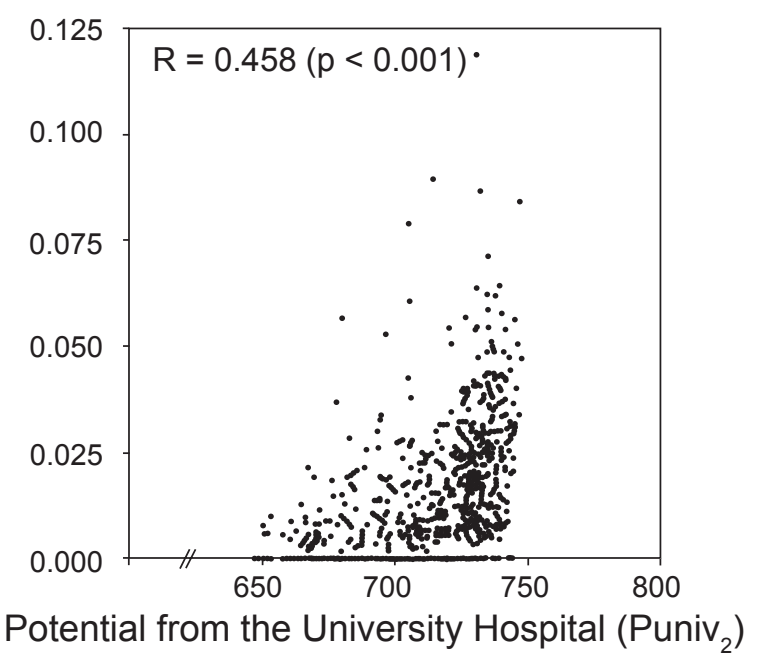

B Admission rate of group B patients

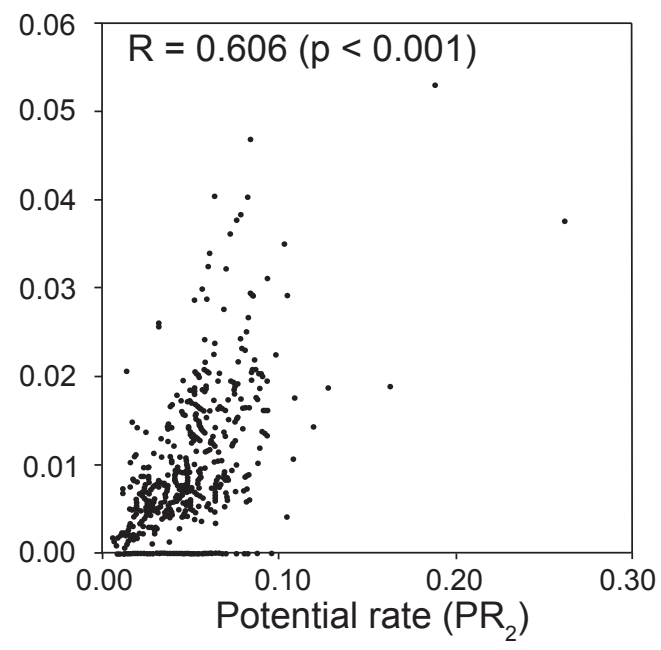

Figure 3 Correlation between admission rate in each mesh and attractive strength of hospitals to the mesh. (A) Admission rate of patients with group A Diagnostic Procedure Combinations was the most associated with the attractive strength from Niigata University Hospital $\left(P_{\text {univ }}\right)(R=0.458, p<0.00 I)$. Each point in the plot corresponds to data on each mesh region. (B) Admission rate of patients with group $B$ Diagnostic Procedure Combinations was the most associated with the ratio between the attractive strength from Niigata University Hospital and that from the enamouring general hospitals $\left(P R_{2}\right)(B, R=0.606, p<0.00 I)$.

to acquire patients who need secondary care. GIS analysis clearly showed that the admission rate of patients who need secondary care in a region was correlated with the ratio of attractive strength of university hospital to the sum of attractive strength of other general hospitals. The potential rate corresponds to the parameter used in Huff's model. Our results suggest that the choice of a hospital from university hospitals and general hospitals for secondary care corresponds to the consumers' spatial behavior proposed in Huff's model. ${ }^{15,16}$ On the other hand, admission rate of patients who need 
Table 4 Results of multiple regression analysis

\begin{tabular}{lllll}
\hline Dependent variables & \multicolumn{4}{c}{ Independent variables } \\
\cline { 2 - 5 } & \multicolumn{5}{c}{$\begin{array}{l}\text { Standard } \\
\text { coefficients }\end{array}$} & $\mathbf{t}$ & sig \\
\hline $\begin{array}{l}\text { Admission rate of } \\
\text { group A patients }\end{array}$ & $P_{\text {univ2 }}$ & 0.435 & $11.25 \mathrm{I}$ & $<0.001$ \\
& $P_{R 2}$ & 0.135 & 3.496 & 0.001 \\
& $P_{\text {univ2 }}$ & 0.085 & 1.785 & 0.075 \\
$\begin{array}{l}\text { Admission rate of } \\
\text { group B patients }\end{array}$ & $P_{R 2}$ & 0.542 & 11.319 & $<0.001$ \\
\hline
\end{tabular}

Dependent variables were admission rates of group $A$ and group $B$ patients, and independent variables were $P_{\text {univ2 }}$ and $P R_{2}$. Multiple regression analysis with forced entry was performed.
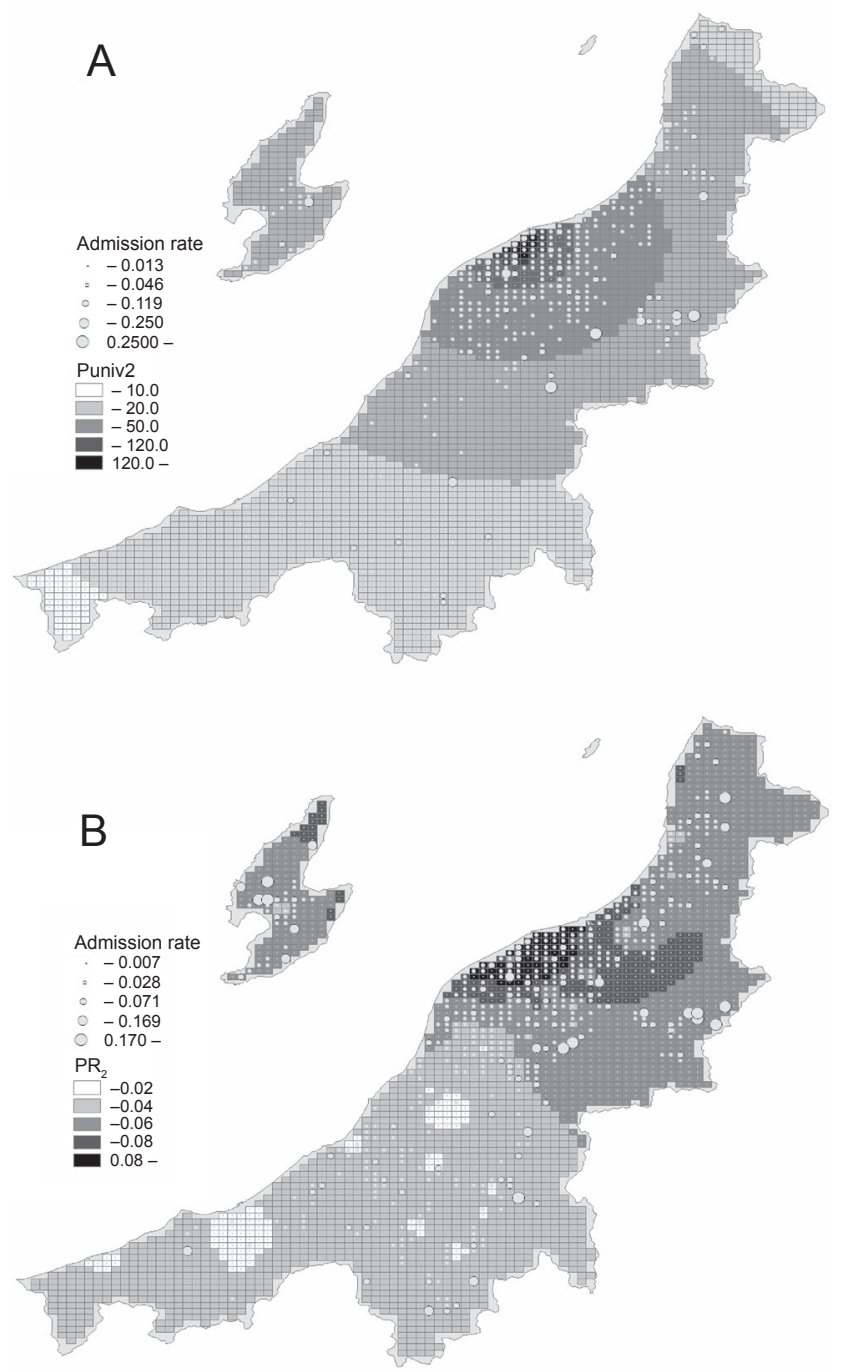

Figure 4 Spatial distribution of admission rates to Niigata University Hospital and attractive strength of hospitals in Niigata Prefecture. (A) The distribution of attractive strength from Niigata University Hospital $\left(\right.$ Puniv $\left._{2}\right)$ was plotted onto the map.The admission rate of group $A$ patients in each mesh region was superimposed on the plot. (B) The distribution of the ratio between the attractive strength from Niigata University Hospital and that from the neighboring general hospitals $\left(P R_{2}\right)$ was plotted onto the map. The admission rate of group $B$ patients in each mesh region was superimposed on the plot. tertiary care in a region was correlated with only attractive strength of university hospital. Therefore, our results suggest that choice of university hospitals is affected by neighboring general hospitals when patients need secondary care but is less affected when patients need tertiary care.

\section{Disclosures}

The author has no conflicts of interest to disclose.

\section{References}

1. Iglehart JK. Japan's medical care system - Part Two. N Engl J Med. 1988;319(17):1166-72.

2. Ikegami N, Campbell JC. Health care reform in Japan: the virtues of muddling through. Health Aff. 1999;18(3):56-75.

3. Iglehart JK. Japan's medical care system. $N$ Engl J Med. 1988;319(12):807-12.

4. Campbell JC, Ikegami N. Long-term care insurance comes to Japan. Health Aff. 2000;19(3):26-39.

5. Organisation for Economic Co-operation and Development (OECD). Health data; 2006.

6. Ikegami N, Campbell JC. Medical care in Japan. N Engl J Med. 1995;333(19):1295-99.

7. Ito M. Health insurance systems in Japan: a neurosurgeon's view. Neurol Med Chir. 2004;44(12):617-28.

8. Tsutsui T, Muramatsu N. Japan's universal long-term care system reform of 2005: containing costs and realizing a vision. $J$ Am Geriatr Soc. 2007;55(9):1458-63.

9. Toyabe S, Kouhei A. Referral from secondary care and to aftercare in a tertiary care university hospital in Japan. BMC Health Serv Res. 2006;6:11.

10. Data from National survey for the impact of introduction of DPCs. Available from: http://www.mhlw.go.jp/shingi/2006/12/s1206-6.html.

11. Nuttall M, van der Meulen J, Emberton M. Charlson scores based on ICD-10 administrative data were valid in assessing comorbidity in patients undergoing urological cancer surgery. J Clin Epidemiol. 2006;59(3):265-73.

12. Sundararajan V, Henderson T, Perry C, Muggivan A, Quan H, Ghali W. New ICD-10 version of the Charlson comorbidity index predicted in-hospital mortality. J Clin Epidemiol. 2004;57(12):1288-94.

13. Wong DWS, Lee J. Statistical analysis of geographic information.New Jersy. John Wiley and Sons, Inc.; 2005.

14. Green MB. Market potential maps of retail sales for the United States. The Great Lakes Geographer. 2000;7(1):53-4.

15. Huff DL. A Probabilistic analysis of shopping center trade areas. Land Econ. 1963;39:81-90.

16. Huff DL. Parameter estimation in the Huff model. ArcUser. 2003; October-December:34-36.

17. Tanser F, Gijsbertsen B, Herbst K. Modelling and understanding primary health care accessibility and utilization in rural South Africa: an exploration using a geographical information system. Soc Sci Med. 2006;63(3):691-705.

18. Fushimi K, Hashimoto H, Imanaka Y, et al. Functional mapping of hospitals by diagnosis-dominant case-mix analysis. BMC Health Serv Res. 2007;7:50.

19. Data from Niigata Prefecture. Available from: http://www.pref.niigata. lg.jp/fukushihoken/1210698060065.html 
